\title{
LA PERCEPCIÓN REMOTA EN EL SEGUIMIENTO DE PLUMAS DE DETRITOS EN BAHÍA DE BANDERAS, JALISCO-NAYARIT, MÉXICO
}

\author{
Remote perception applied to the following of detritus plumes in Bahía de Banderas, Jalisco-Nayarit, Mexico
}

\author{
Ignacio Omar MIRELES LOERA ${ }^{1 *}$, Anatoliy FILONOV ${ }^{2}$, \\ Carlos Alberto GONZÁLEZ DE LUNA ${ }^{2}$ e Irina TERESHCHENKO ${ }^{2}$
}

${ }^{1}$ Centro Universitario de la Costa, Universidad de Guadalajara, Av. Universidad 203, Delegación Ixtapa, 48280 Puerto Vallarta, Jalisco, México

${ }^{2}$ Posgrado en Ciencias en Hidrometeorología, Universidad de Guadalajara. Blvd. Marcelino García Barragán 1421, Col. Universitaria, 44430. Guadalajara, Jalisco, México

*Autor para correspondencia: mireles.ceo@gmail.com

(Recibido enero 2018; aceptado octubre 2018)

Palabras clave: clasificación no supervisada, colorimetría, Landsat, Modis

\section{RESUMEN}

La percepción remota es una alternativa muy eficiente para el monitoreo de plumas de detritos y de contaminantes orgánicos. Las técnicas de colorimetría y métodos de clasificación no supervisada perfilan estas plumas, las cuales se pueden vectorizar y así trazar su evolución respecto al tiempo y al espacio. Lo anterior, en la práctica, abarata el costo de investigación y permite tener una mayor cobertura espacial y temporal. Este trabajo presenta el caso de estudio de Bahía de Banderas, Jalisco-Nayarit, México, donde se estudió el vertido de detritos por el río Ameca en el periodo 2006-2014 a través de un producto combinado de imágenes Landsat y Modis.

Key words: non-supervised classification, colorimetry, Landsat, Modis

\begin{abstract}
Remote sensing is a very efficient alternative with regard to the monitoring of detritus plumes and organic pollutants. Colorimetry and unsupervised classification methods outline these plumes, which can be vectorized and their evolution traced with respect to time and space. In practice, this lowers the cost of research and allows for greater spatial and temporal coverage. This work presents the case study of Bahía de Banderas, Jalisco-Nayarit, Mexico, where the detritus discharge by the Ameca river in the period 2006-2014 was studied through a combined product of Landsat and Modis images.
\end{abstract}

\section{INTRODUCCIÓN}

Las observaciones del color del océano tienen gran potencial para estudiar la conectividad entre litorales y zonas costeras mediante el rastreo de la dispersión de las plumas vertidas por los ríos. La identificación de estas plumas, las variaciones en su extensión, patrones de dispersión y tasas de mezcla 
con aguas oceánicas es crítica en todos los aspectos de la oceanografía regional y de la plataforma continental (Lohrenz et al. 1990).

Los procesos físicos que caracterizan la dinámica de las bahías afectan de manera notable e incluso determinante (o interactúan con) procesos oceanográficos de índole química, geológica y biológica que tienen lugar en estos cuerpos de agua. Por ello, su descripción y análisis es indispensable para abordar y resolver problemas concernientes a cada una de las ramas de la oceanografía costera (Plata 2007).

Actualmente, la determinación de concentraciones de material suspendido de forma global y precisa con datos de sensores remotos sigue siendo un reto. Sin embargo, se han logrado buenas aproximaciones en aplicaciones específicas con datos provenientes de una variedad de sensores de diversas precisiones radiométricas y resoluciones espaciales y temporales, como los transportados a bordo de los satélites Landsat, Spot, NOAA, SeaStar, Aqua, Terra, entre otros (Acker et al. 2002).

Así pues, la teledetección ha demostrado tener un potencial elevado en el monitoreo y seguimiento de fenómenos como la dispersión de residuos y contaminantes. La percepción remota de recursos naturales se basa en un sistema de adquisición de datos a distancia sobre la superficie terrestre, basado en las propiedades de la radiación electromagnética y en su interacción con los materiales de la superficie terrestre (Pérez 2012).

El estudio de la dinámica de los procesos de transporte en las aguas de la Bahía de Banderas, Jalisco-Nayarit, México, es de importancia fundamental para la planeación de estrategias que permitan el desarrollo sustentable de esta región turística y la adecuada construcción de obras de protección costera como espigones y rompeolas.

En las últimas décadas, el agua de Bahía de Banderas ha sido impactada por contaminantes debido al crecimiento de descargas incontroladas de aguas negras de hoteles y zonas habitacionales en Puerto Vallarta, Nuevo Vallarta y los alrededores de la bahía. La planificación adecuada de las medidas para eliminar la mayor contaminación posible de la bahía es necesaria no sólo por su gran impacto económico, sino también para conocer la zona de mayor concentración de contaminantes y su distribución espacial y temporal (Plata et al. 2006).

La concentración del total de contaminantes y sedimentos suspendidos provenientes del lavado continental y materia orgánica (detritos), constituye uno de los parámetros de calidad del agua más importantes (Rodríguez y Gilbes 2009), principalmente porque se relaciona con la producción y flujo de metales pesados y microcontaminantes (Ekercin 2007).

Estos sedimentos consisten en partículas suficientemente finas para ser transportadas en suspensión por el agua en movimiento (Campbell 2007), como las de arcilla, limo y arena. Además de afectar la calidad del agua, los detritos son indicativos de problemas de erosión en las cuencas hidrográficas. Más aún, el estudio de esta materia, principalmente en regiones costeras, tiene una gran importancia ecológica. Las altas concentraciones de detritos en zonas costeras pueden afectar la productividad del fitoplancton y de la vegetación acuática sumergida, el crecimiento de corales, la dinámica de nutrientes, y el transporte de contaminantes y otros materiales (Miller y Mckee 2004).

Desde finales la década de 1970, se han realizado estudios de transporte de sedimentos suspendidos (TSS) utilizando datos de plataformas satelitales (Ritchie y Cooper 2001) cuyos sensores miden la cantidad de radiación solar reflejada por la superficie del agua a diferentes longitudes de onda. Actualmente el TSS es uno de los parámetros que se mide con más éxito por medio de la percepción remota (Ekercin 2007).

En lo que respecta al estudio de contaminantes en Bahía de Banderas, destacan los trabajos de Kelly et al. (2010a, b), que presentan un estudio sistemático de más de 30 puntos de muestreo durante varias temporadas. En estos trabajos se concluye que los procesos que determinan la composición y calidad del agua en Bahía de Banderas son el aporte de contaminantes, particularmente de materia fecal, así como de agua dulce por los ríos, lo que se evidencia por la disminución de la salinidad acompañada por el aumento en la concentración de nutrientes.

\section{MATERIALES Y MÉTODOS}

\section{Área de estudio}

El área de estudio (Fig. 1a) es Bahía de Banderas, que se localiza en la costa occidental de México y constituye el límite sur del Golfo de California en su parte continental, abarcando parte de los estados de Jalisco y Nayarit. Geográficamente, la bahía está ubicada entre los $20^{\circ} 25^{\prime}$ y $20^{\circ} 47^{\prime}$ de latitud norte y los $105^{\circ} 41^{\prime}$ y $105^{\circ} 25^{\prime}$ de longitud oeste.

Si se delimita la bahía mediante una línea imaginaria desde punta de Mita, Nayarit, en el norte, hasta Cabo Corrientes, Jalisco, en el sur, su área es de aproximadamente $1200 \mathrm{~km}^{2}$, con un volumen de $3.68 \times 10^{2} \mathrm{~km}^{3}$. 

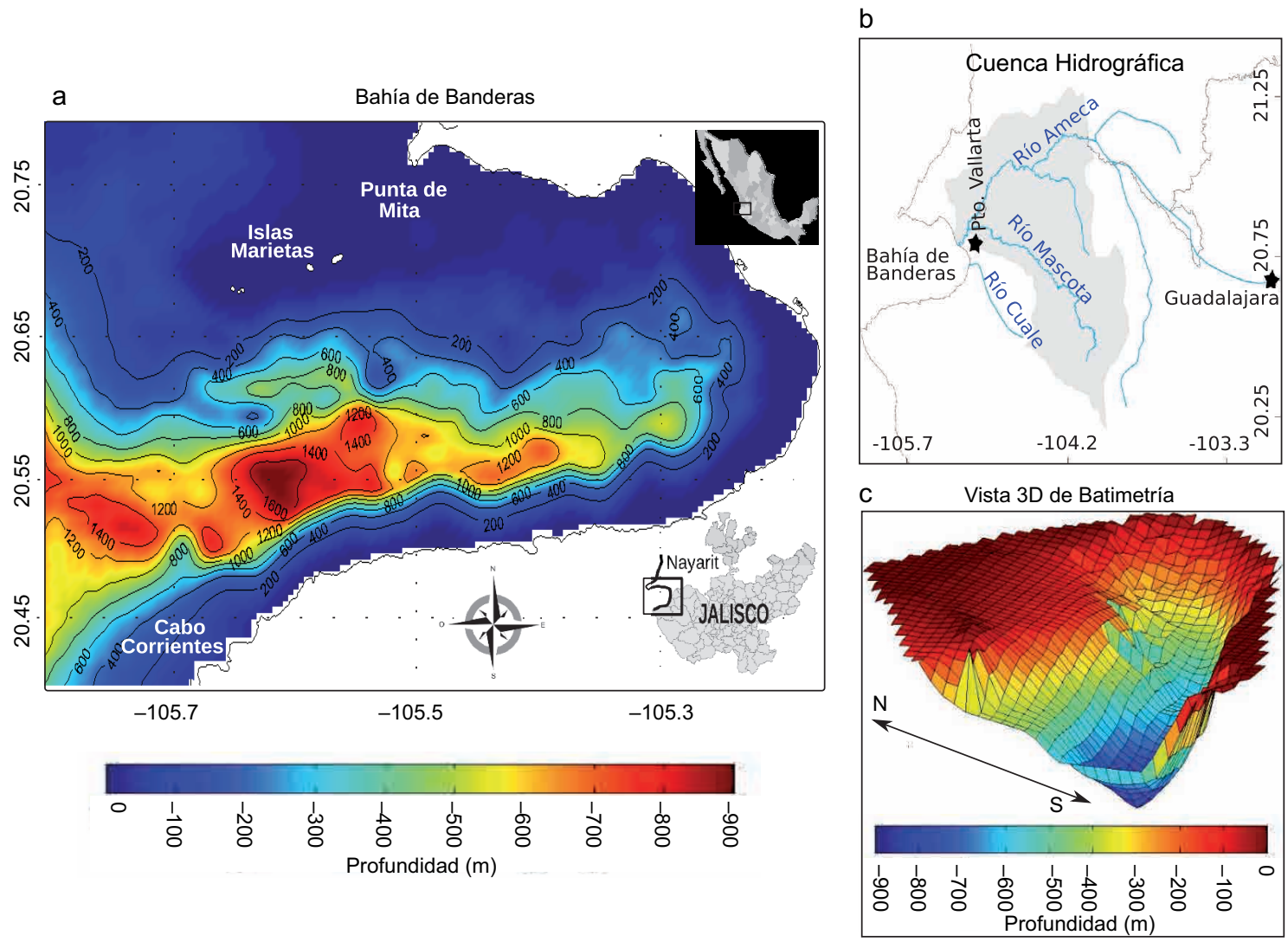

Fig. 1. (a) Ubicación y batimetría de Bahía de Banderas, (b) cuenca hidrológica, (c) vista en 3D de la batimetría de Bahía de Banderas

La bahía tiene un ancho promedio (norte-sur) de $30 \mathrm{~km}$ y una longitud promedio (este-oeste) de 40 $\mathrm{km}$. Al sureste, Bahía de Banderas está rodeada de montañas con una altura máxima de $1500 \mathrm{~m}$. Al norte existen lomas con elevaciones que van de 500 a 750 $\mathrm{m}$. Entre las montañas del este y las lomas del norte se halla un valle DE alrededor de $15 \mathrm{~km}$ de ancho, a través del cual fluye el río Ameca (Fig. 1b), que es el más grande de la región y desemboca unos $10 \mathrm{~km}$ al norte de la ciudad de Puerto Vallarta (Plata 2007).

Al noroeste de Bahía de Banderas y aproximadamente a $10 \mathrm{~km}$ de Punta de Mita se encuentra el archipiélago de las Islas Marietas, un conjunto de islas pequeñas, islotes y bajos con una extensión de $8.3 \mathrm{~km}$. Al suroeste de la bahía, se halla el grupo de islotes conocido como Los Arcos, que ocupa un área aproximada de $0.3 \mathrm{~km}^{2}$ (Medina-Rosas 1997).

La profundidad máxima de la bahía es de $1436 \mathrm{~m}$ (INEGI-SPP 1983) y su profundidad promedio es de $273 \mathrm{~m}$. La pendiente relativa del fondo en la parte norte, con un valor promedio de 0.012 , es mucho menor que en la parte sur, con un valor promedio de 0.080 . Hacia el centro de la bahía, a unos $8 \mathrm{~km}$ de la costa sur, se localiza un cañón profundo cuyo eje mayor está orientado aproximadamente en dirección este-oeste y se extiende hasta el extremo este de la bahía (Fig. 1c). Al noroeste de la bahía, en la zona comprendida entre las islas Marietas y Punta de Mita, la profundidad es menor a $25 \mathrm{~m}$ y existe un banco de arena sumergido que no permite el libre intercambio de masas de agua entre la parte noroeste de este cuerpo de agua y el mar abierto (Plata 2007).

En la bahía confluyen tres sistemas de corrientes oceánicas: la de California, de masas de agua fría y de baja salinidad, que fluye hacia el sur desde Baja California; la de Costa Rica, que posteriormente se convierte en la Corriente Norecuatorial, de masas de agua caliente, salinidad intermedia y que fluye hacia el norte desde el sureste de México (Baumgartner y Christiensen 1985), y la Corriente Costera Mexicana, que fluye hacia el norte desde el sureste de México. Además, la bahía tiene un aporte de aguas cálidas y de alta salinidad del Golfo de California. La convergencia de estas corrientes da lugar a cambios de salinidad, temperatura y patrones de circulación estacional en la región (Álvarez y Gaitán 1994). 
En la costa de Bahía de Banderas se localizan 32 cuerpos de agua (ríos, arroyos, esteros y lagunas) y numerosos escurrimientos temporales (127), ubicados en su mayoría en las zonas centro y sur. En la bahía desembocan los ríos Ameca, Pitillal, Cuale, Los Horcones, Mismaloya, Tuito y La Puerta, que tienen caudal la mayor parte del año, además de los arroyos temporales Los Coamiles, Pantoque, El Burro, El Carrizal, Caloso, La Cumbre, Hondo, Palo María, Amapas, El Salto, Camarones, El limón, Pilidad, La Cueva, Chocota, Tecomata, Pizota, Coquitos, Majahuitas, Maxeque y Tabo (Plata 2007).

El río Ameca nace en el Bosque de la Primavera, a escasos $23 \mathrm{~km}$ al oeste de la ciudad de Guadalajara y discurre en dirección oeste. Toma su nombre de la ciudad de Ameca que atraviesa y tiene como afluentes principales los ríos Ahuacatlán y Amatlán de Cañas. El río Los Horcones es alimentado por las aguas que discurren de las montañas en el municipio de Cabo Corrientes, en tanto que el río Cuale (el cual atraviesa el pueblo minero El Cuale) tiene dos afluentes principales que nacen en lo alto de la sierra del Cuale (también llamada sierra del Tuito) a una altura de $2740 \mathrm{~m}$ en las montañas de Talpa de Allende (Fig. 1b).

Si bien es cierto que la bahía de estudio tiene presencia de muchas entradas de agua dulce, en este trabajo sólo se analiza el escurrimiento del río Ameca, ya que es uno de los escurrimientos más importantes que desembocan en Bahía de Banderas.; su cuenca tiene una extensión territorial de $12255 \mathrm{~km}^{2}$ y una precipitación normal anual (en el periodo 19712000) de $1020 \mathrm{~mm}$. Su escurrimiento natural medio superficial total es de $2235 \mathrm{hm}^{3} /$ año (Gonzalez-de Luna 2017).

\section{Bases de datos}

Para el análisis del transporte de residuos dentro de la bahía se formó un producto combinado de escenas Landsat $(5,7$ y 8$)$ correspondientes a los sensores MSS/TM, ETM+ y OLI/TIRS, respectivamente, y escenas Modis de un periodo de nueve años (20062014) (Cuadro I).

Es importante mencionar que los satélites Landsat tienen un periodo de paso de 16 días, existiendo un promedio de dos escenas por mes en los años que se encuentran en operación los satélites Landsat 5 y 7 y un promedio de tres escenas por mes en los años en que inició la operación del satélite Landsat 8. La resolución espacial del sensor a bordo de Landsat 5 es de $30 \mathrm{~m}$ para la multiespectral y de $120 \mathrm{~m}$ para la termal; la del sensor a bordo de Landsat 7 es de $30 \mathrm{~m}$ para la multiespectral, $60 \mathrm{~m}$ para la termal y

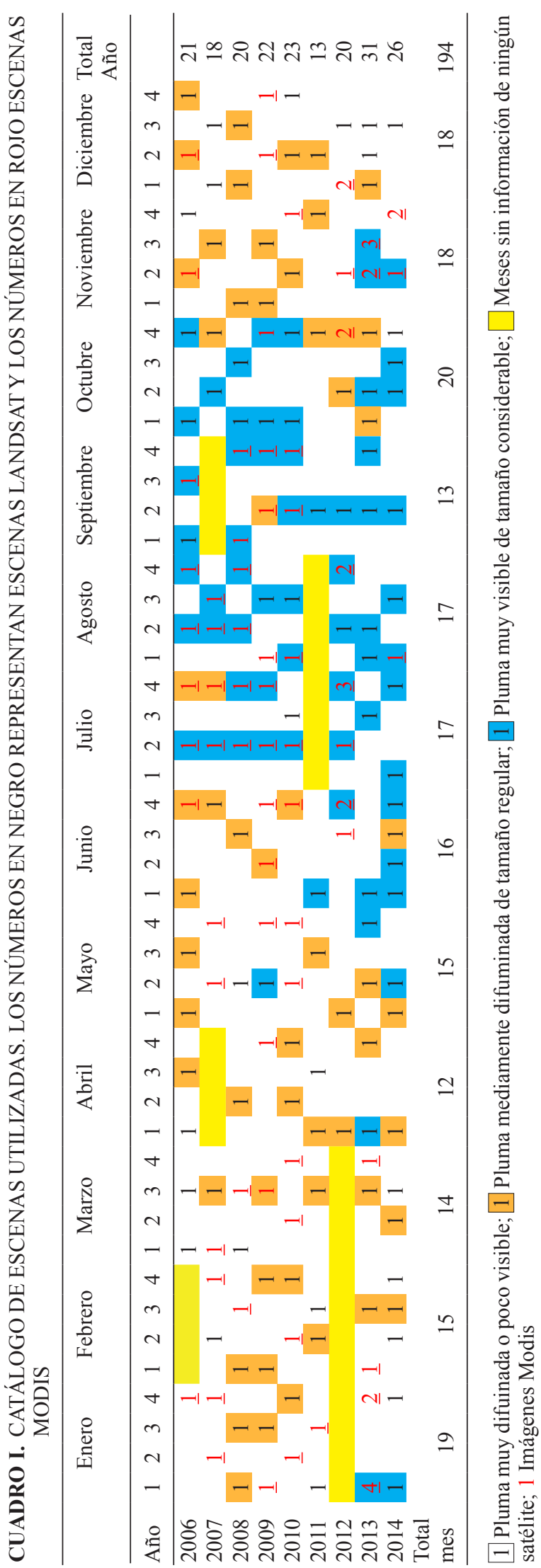


$15 \mathrm{~m}$ para la pancromática, finalmente la resolución espacial del sensor a bordo del satélite Landsat 8 es de $30 \mathrm{~m}$ para la multiespectral, $100 \mathrm{~m}$ para la termal y $15 \mathrm{~m}$ para la pancromática.

Por otra parte, las escenas Modis sólo contienen las tres bandas con resolución aceptable y corresponden al espectro visible. Están codificadas a 8 bits y tienen una resolución espacial de $250 \mathrm{~m}$. Estos satélites tienen un periodo de paso de 2 días.

\section{Análisis realizados}

Con relación al procesamiento de información, se utilizó el Sistema de Información Geográfica (SIG) ArcView/ArcGis empleando los módulos de procesamiento Ráster y Multivariable (Raster Processing and Multivariate). Para el tratamiento de matrices se utilizó el programa Matlab. Se analizaron un total de 194 imágenes de satélites, 85 del satélite Modis y 109 del satélite Landsat. De las 109 imágenes Landsat, seis corresponden a Landsat 5, 84 a Landsat 7 y 19 a Landsat 8.

Debido a las características de la resolución espacial y temporal mencionadas con anterioridad, se les dio preferencia a las escenas Landsat. Se eliminaron aquellas con presencia excesiva de nubes o detección dudosa de plumas de detritos, y se sustituyeron por escenas del satélite Modis para tener al menos dos escenas por mes en dicho periodo de estudio. Posteriormente se realizó el tratamiento y armado de las bandas por cada escena y se validaron sus características.

Es importante señalar que que el sensor del satélite Landsat 7 presenta un error en el corrector de la línea de escáner (scan line corrector), por lo que se estimaron los límites de cada categoría de las zonas sin información visualizando la tendencia con apoyo de las franjas visibles. Afortunadamente el ancho de las bandas sin información no es lo suficientemente grande como para imposibilitar la tendencia del límite de la categoría identificada. Además, mucha de la información faltante en las franjas no visibles se interpoló con información de las imágenes de Modis.

Para la identificación de sedimentos suspendidos en la bahía se utilizó el método de clasificación no supervisada. Esta clasificación muestra mayor eficiencia para detectar diferencias en la concentración de sedimentos suspendidos que la clasificación supervisada. Esto se debe a la dificultad de visualizar en la escena original las diferencias en concentración de sedimentos para darle el entrenamiento adecuado a la herramienta de clasificación supervisada (Pérez y Nadja 2011).
Para extraer las plumas de detritos suspendidos (Fig. 2a) se eliminó de cada escena la superficie continental, utilizando como límite de costa el límite estatal 2010 proporcionado por el Instituto Nacional de Estadística y Geografía (INEGI) con un área de influencia acorde con el tamaño de pixel de cada imagen, para garantizar la no interferencia de la porción continental en la clasificación. Una vez que sólo se tuvo la parte de agua costera en las escenas, por medio de la técnica de clasificación no supervisada se creó una máscara de filtrado con base a todos aquellos pixeles con alta reflectividad correspondientes a zonas cubiertas con nubes para posteriormente extraerlos de la imagen (Fig. 2b).

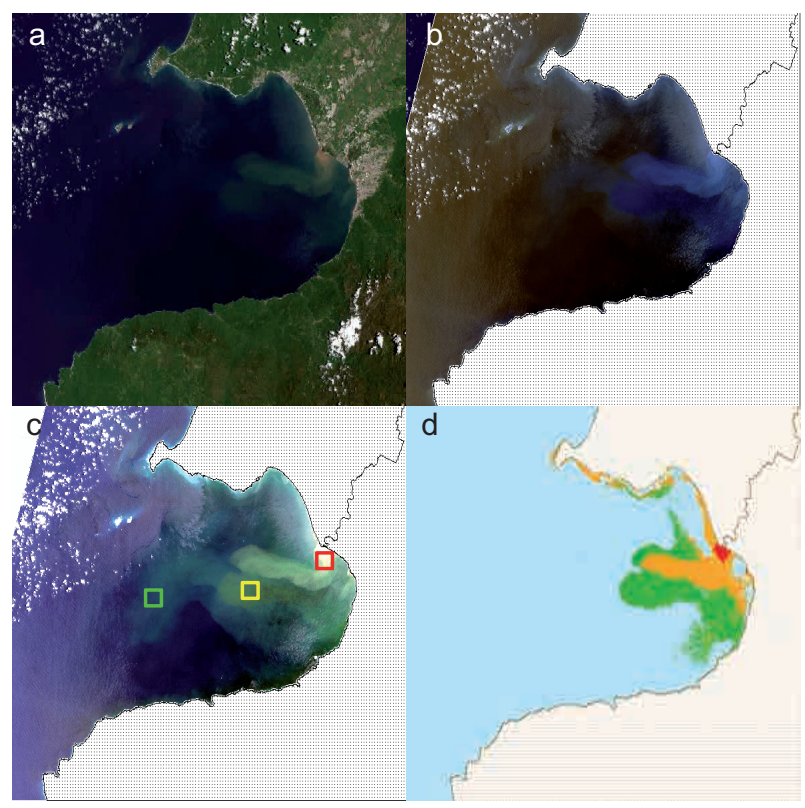

Fig. 2. (a) Escena obtenida del satélite Landsat 8; (b) escena obtenida después de procesarla quitando la parte continental y eliminando las nubes; (c) escena obtenida al someterla a un procesamiento de clasificación no supervisada, donde los recuadros de color rojo, amarillo y verde representan los criterios utilizados para la vectorización de la pluma de detritos, y (d) plumas vectorizadas en clases

La finalidad de eliminar la porción continental y de nubes de una escena es que el algoritmo de clasificación sólo considere las zonas que nos interesan, correspondientes a la parte de agua, dando como resultado una mayor identificación de las diferencias de reflectividad, lo cual es muy importante para la correcta identificación de las plumas.

Una vez con la escena sin la parte continental y sin el error radiométrico que causarían las nubes, se 
corrió nuevamente el algoritmo de la clasificación no supervisada utilizando las bandas 1, 2 y 3 , las cuales corresponden al espectro visible $(0.45$ a $0.69 \mu \mathrm{m})$. Las bandas 4, 5, 6 y 7 corresponden al infrarrojo cercano $(0.76$ a $0.90 \mu \mathrm{m})$, al infrarrojo lejano $(1.55$ a $1.75 \mu \mathrm{m})$, al infrarrojo térmico lejano (10.4 a 12.5 $\mu \mathrm{m})$ y al infrarrojo térmico medio $(2.08 \mathrm{a} 2.35 \mu \mathrm{m})$, respectivamente, del satélite Landsat-5. En el satélite Landsat-7 las bandas 1, 2 y 3 corresponden al espectro visible $(0.45$ a $0.69 \mu \mathrm{m})$, en tanto que las bandas 4 y 5 al infrarrojo cercano $(0.78$ a $0.90 \mu \mathrm{m})$ y al infrarrojo lejano $(1.55$ a $1.75 \mu \mathrm{m})$, respectivamente. Las bandas 6 y 7 corresponden al infrarrojo térmico lejano $(10.4 \mathrm{a} 12.5 \mu \mathrm{m})$ y la banda 8 al infrarrojo térmico medio (2.09 a $2.35 \mu \mathrm{m})$.

En el satélite Landsat 8 la banda 1 corresponde a la azul profundo para estudios costeros de aerosol $(0.433$ a $0.453 \mu \mathrm{m})$, las 2,3 y 4 se asocian con el espectro visible $(0.45$ a $0.68 \mu \mathrm{m})$, la 5 con el infrarrojo cercano $(0.845$ a $0.885 \mu \mathrm{m})$, la 6 con el infrarrojo lejano $(1.56$ a $1.66 \mu \mathrm{m})$, la 7 con el infrarrojo térmico medio $(2.1$ a $2.3 \mu \mathrm{m})$, la 8 con la banda de cirrus (1.36 a $1.39 \mu \mathrm{m})$, la 9 con el infrarrojo térmico lejano $(10.6 \mathrm{a} 11.2 \mu \mathrm{m})$ y la $10 \mathrm{con}$ el infrarrojo térmico lejano II (11.5 a 12.5). Ya que tanto la clasificación de escenas como la selección de los puntos que se utilizaron para determinar el nivel de concentración de los detritos se realizó hasta ese momento de manera visual, se procedió a vectorizar y separar las matrices correspondiente a las plumas de detritos suspendidos para analizarlas con independencia en el programa Matlab en cuanto su dispersión, concentración y desplazamiento. (Fig. 2c).

Una vez procesadas las imágenes con un algoritmo de clasificación no supervisada, se determinaron los mínimos y máximos del comportamiento espectral en cada categoría, con la intención de establecer las fronteras radiométricas de cada categoría y tener un criterio estándar para separar la clase correspondiente a la pluma en categorías según su concentración de detritos (Fig. 2d).

Para calibrar y homogeneizar las categorías entre distintos sensores se utilizaron imágenes en las que se visualiza perfectamente lo correspondiente a la categoría 5 (alto o concentrado) para clasificarla. Con base en esta primera aproximación se extrapolaron las otras cuatro categorías. Lo anterior se complementó con escenas de distintos sensores, pero de fechas cercanas, en las que se establecieron los límites de una zona de pluma que por su cercanía temporal correspondiera a la misma categoría en cada imagen.

\section{RESULTADOS}

Debido a que la concentración de plumas de detritos no es homogénea en toda su extensión tanto espacial como temporal, se generaron varias clases según su concentración que van desde disperso (categoría 1) hasta muy denso (categoría 3), utilizándose para la primera delimitación un $10 \%$ de las imágenes con mayor presencia de plumas. Sobre estas escenas se definieron de manera visual las zonas extremas de concentración de detritos, entre las cuales la desembocadura del río Ameca representa la zona de mayor concentración de detritos, y el centro de la bahía la zona donde los detritos suspendidos están más dispersos (lo que se fortaleció por presentar plumas que generalmente sólo se pueden apreciar con un realce del falso color).

Una vez procesadas el $100 \%$ de las escenas se calcularon las estadísticas del comportamiento espectral de cada categoría. Ya que las escenas corresponden a diferentes días y todas contaban con diferente cantidad de nubosidad, se determinaron los límites de cada categoría tomando en cuenta el límite inferior y superior de su respuesta espectral, estableciéndose a su vez un promedio central en base a esos límites (Cuadro II para Modis y Cuadro III para Landsat).

Es importante mencionar que el propio algoritmo de clasificación no supervisada dividió la pluma en varias categorías que generalmente coincidían con las categorías establecidas en los rangos radiométricos sin importar a cuál sensor correspondían.

Para una mayor conceptualización y sustento de las tres categorías, se comparó la respuesta espectral de las plumas respecto a la de tres superficies conocidas,

CUADRO II. VARIACIÓN DE LOS PROMEDIOS DE LOS LÍMITES ESPECTRALES POR CATEGORÍA EN LAS ESCENAS MODIS

\begin{tabular}{lllll}
\hline \multirow{2}{*}{ Categoría } & \multicolumn{4}{c}{ MODIS } \\
\cline { 2 - 5 } & Banda & $\begin{array}{c}\text { Promedio } \\
\text { general }\end{array}$ & $\begin{array}{c}\text { Promedio } \\
\text { inferior }\end{array}$ & $\begin{array}{c}\text { Promedio } \\
\text { superior }\end{array}$ \\
\hline \multirow{3}{*}{1} & Rojo & 25.36 & 10.08 & 32.65 \\
& Verde & 40.51 & 32.73 & 48.3 \\
& Azul & 36.72 & 30.05 & 43.39 \\
\hline \multirow{3}{*}{2} & Rojo & 44.01 & 34.38 & 53.64 \\
& Verde & 58.01 & 49.1 & 66.92 \\
& Azul & 48.3 & 40.34 & 56.26 \\
\hline \multirow{3}{*}{3} & Rojo & 83.49 & 57.51 & 109.46 \\
& Verde & 83.72 & 66.89 & 100.54 \\
& Azul & 60 & 46.76 & 73.24 \\
\hline
\end{tabular}


CUADRO III. VARIACIÓN DE LOS PROMEDIOS DE LOS LÍMITES ESPECTRALES POR CATEGORÍA Y BANDA DE LOS SENSORES LANDSAT 5, 7 Y 8

\begin{tabular}{|c|c|c|c|c|c|c|c|c|c|c|}
\hline \multirow[b]{2}{*}{ Categoría } & \multirow[b]{2}{*}{ Banda } & \multicolumn{3}{|c|}{ Landsat-5 } & \multicolumn{3}{|c|}{ Landsat-7 } & \multicolumn{3}{|c|}{ Landsat-8 } \\
\hline & & $\begin{array}{c}\text { Promedio } \\
\text { general }\end{array}$ & $\begin{array}{l}\text { Promedio } \\
\text { inferior }\end{array}$ & $\begin{array}{l}\text { Promedio } \\
\text { superior }\end{array}$ & $\begin{array}{c}\text { Promedio } \\
\text { general }\end{array}$ & $\begin{array}{l}\text { Promedio } \\
\text { inferior }\end{array}$ & $\begin{array}{c}\text { Promedio } \\
\text { superior }\end{array}$ & $\begin{array}{c}\text { Promedio } \\
\text { general }\end{array}$ & $\begin{array}{l}\text { Promedio } \\
\text { inferior }\end{array}$ & $\begin{array}{c}\text { Promedio } \\
\text { superior }\end{array}$ \\
\hline \multirow{3}{*}{1} & $\begin{array}{l}\text { Infrarrojo } \\
\text { cercano }\end{array}$ & 12.92 & 10.83 & 15 & 14 & 12.72 & 15.28 & 5861.76 & 5664.71 & 6058.82 \\
\hline & Rojo & 21.08 & 18.67 & 23.5 & 32.77 & 28.65 & 36.9 & 6852.94 & 6476.47 & 7229.41 \\
\hline & Verde & 21.08 & 18.17 & 24 & 47.71 & 43.26 & 52.16 & 8032.35 & 7617.65 & 8447.06 \\
\hline \multirow{3}{*}{2} & $\begin{array}{l}\text { Infrarrojo } \\
\text { cercano }\end{array}$ & 16.5 & 14.5 & 18.5 & 16.34 & 14.71 & 17.97 & 6685.71 & 6264.29 & 7107.14 \\
\hline & Rojo & 26.13 & 23.75 & 28.5 & 42.18 & 36.7 & 47.67 & 8242.86 & 7514.29 & 8971.43 \\
\hline & Verde & 31.38 & 27.75 & 35 & 57.43 & 51.85 & 63.01 & 9196.43 & 8714.29 & 9678.57 \\
\hline \multirow{3}{*}{3} & $\begin{array}{l}\text { Infrarrojo } \\
\text { cercano }\end{array}$ & 22.17 & 18.33 & 26 & 23.41 & 17.32 & 29.5 & 8131.25 & 7237.5 & 9025 \\
\hline & Rojo & 39.33 & 34.33 & 44.33 & 68.73 & 53.14 & 84.32 & 10212.5 & 9712.5 & 10712.5 \\
\hline & Verde & 42.5 & 38.33 & 46.67 & 77.36 & 67.75 & 86.96 & 10362.5 & 10050 & 10675 \\
\hline
\end{tabular}

que pueden estar relacionadas con la constitución de las plumas de detritos suspendidos en la bahía. La primera es la superficie del escurrimiento dentro del continente en los meses con mayor presencia de plumas (junio-septiembre), la segunda es la reflectividad de la playa contigua a la desembocadura del río, y la tercera corresponde al suelo desnudo y con materia orgánica aledaño al escurrimiento dentro del continente. Del análisis anterior se derivó que la pluma clasificada como de categoría 3 (muy densa) muestra una firma similar a la del escurrimiento pero difiriendo en la cantidad de infrarrojo cercano absorbido. lo cual probablemente se deba a la mayor profundidad de la columna de agua en la bahía que en el cauce; en este análisis la categoría 3 emite cerca del $70 \%$ del infrarrojo emitido por el cauce.

\section{Promedio de concentración de detritos}

Después de clasificar las plumas respecto a su nivel de concentración parametrizada de detritos, se procedió a determinar el promedio mensual del periodo 2006-2014, así como la superficie promedio que ocupan por mes (Fig. 3). Ya que en algunos meses presentan una diferencia muy marcada entre sus plumas de detritos, se señala con un código de color en líneas negras la zona donde la coincidencia es menor al $20 \%$ de plumas, la cual se ve reflejada en plumas de categoría 1; esto con la intención de establecer una zona más pequeña con un nivel de coincidencia del $80 \%$, lo que resulta ser una marca mucho más representativa del periodo de tiempo analizado.

\section{Comparación de la respuesta espectral de las clases respecto a otras superficies conocidas}

Para analizar la respuesta espectral de la pluma de detritos se realizaron transectos cerca de la línea costera (Fig. 4) en cuatro escenas obtenidas con el sensor Landsat 8, y los espectros obtenidos se compararon con las firmas espectrales de la clorofila, algas, materia orgánica y agua turbia.

Aunque cada escena cuenta con 10 bandas, sólo se trabajaron para este análisis las correspondientes al espectro visible (banda 2, 3 y 4 ) y al infrarrojo cercano e infrarrojo medio (bandas 5 y 6 , respectivamente) debido a que estas bandas muestran la mayor respuesta de los sedimentos suspendidos detectados en las imágenes, coincidiendo también con las bandas empleadas para la detección de clorofila, sedimentos y materia orgánica (cuadros IV a VII). Los cuadros muestran que el valor de la firma espectral sobre el agua de mar con concentración de categoría 2 tiene mayor reflectividad en el verde, seguido por el azul y el rojo. Sin embargo, aunque el verde y el azul predominan, se observa que el rojo tiene un crecimiento más marcado conforme aumenta la concentración de partículas suspendidas. Se puede observar también que la reflectividad del infrarrojo cercano e infrarrojo medio empiezan a incrementarse aunque de forma muy lenta, lo que implica la transición a agua turbia. En lo referente a concentraciones correspondientes a la categoría 3 se observa que la mayor respuesta espectral ocurre en el rojo, seguido por el verde, infrarrojo cercano, azul y, finalmente, infrarrojo medio. 

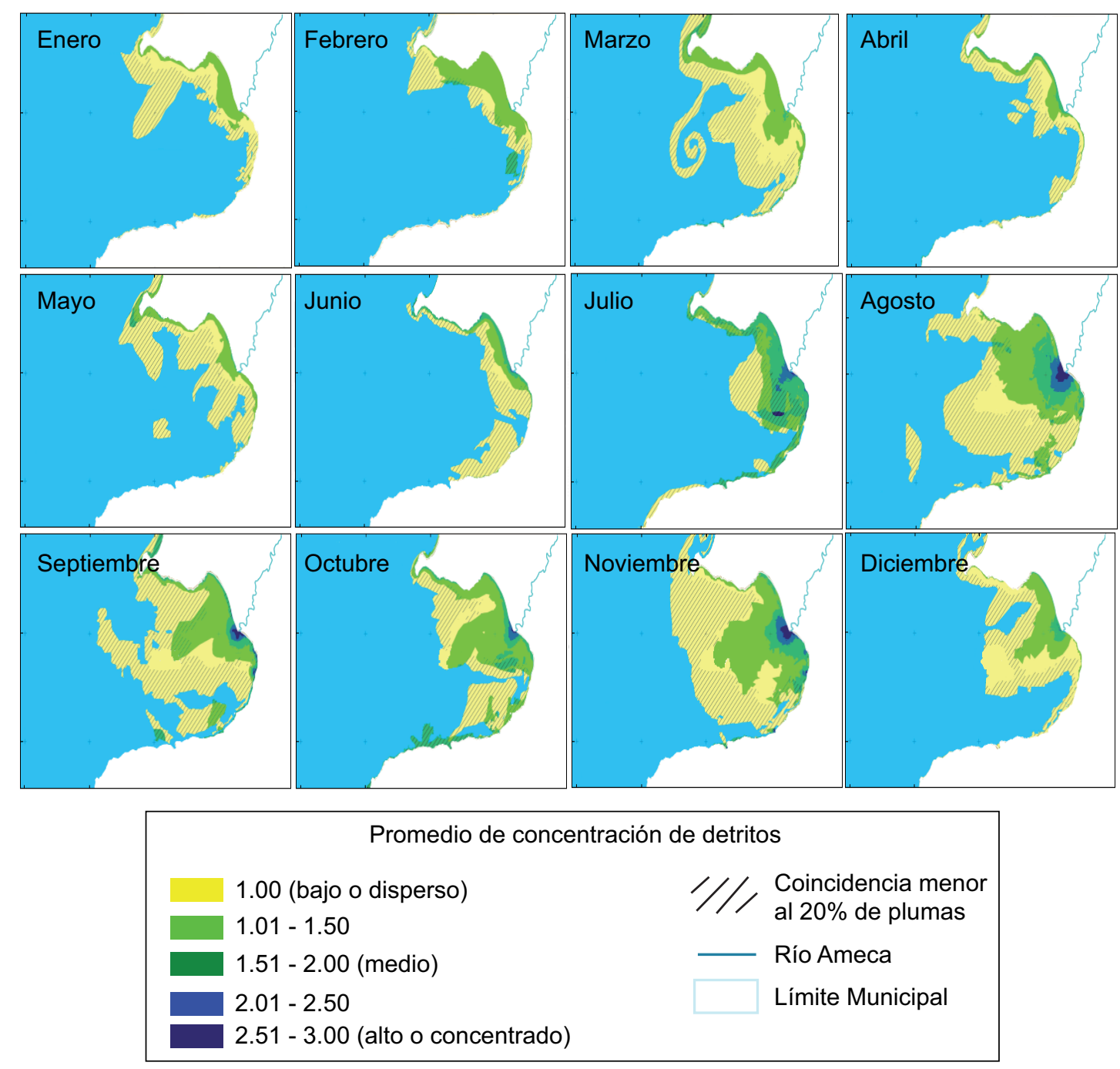

Fig. 3. Plumas de detritos vectorizadas por mes del periodo 2006-2014, donde el color amarillo representa la categoría 1 , correspondiente a las plumas de detritos más bajas y dispersas. El color verde representa la categoría 2, de plumas de detritos de media densidad, y el color azul representa la categoría 3, que comprende las plumas de detritos concentradas

\section{DISCUSIÓN}

La zona de estudio se caracteriza por tener una complicada batimetría, lo que a su vez hace que el estudio de las corrientes sea más difícil. Por este motivo el uso de técnicas de percepción remota aplicadas al estudio de dispersión de plumas de detritos en la capa superficial de las aguas costeras representa un gran avance en el estudio de este tipo de problemas.

Por sí mismo, el uso de escenas obtenidas de los satélites Modis y Landsat otorga mucha información sobre la dispersión de la pluma de detritos; sin embargo, al aplicar un procesamiento para una clasificación no supervisada obtenemos información que estaba oculta en la escena. Si a esta escena le quitamos los elementos que provocan ruido en la radianza se puede afinar mucho más el método de clasificación no supervisada, lo que deriva en la obtención de información sobre las partículas en proceso de sedimentación y capas subsuperficiales.

Con la clasificación anterior se pueden vectorizar las plumas separando por zonas con relación a la densidad de los detritos suspendidos, lo que permite obtener una tendencia clara de su trayectoria e inferir la velocidad de desplazamiento. Sin embargo, es importante aclarar que este trabajo analiza la distribución espacial de los detritos sólo en la capa superficial, por lo que no puede establecerse con 


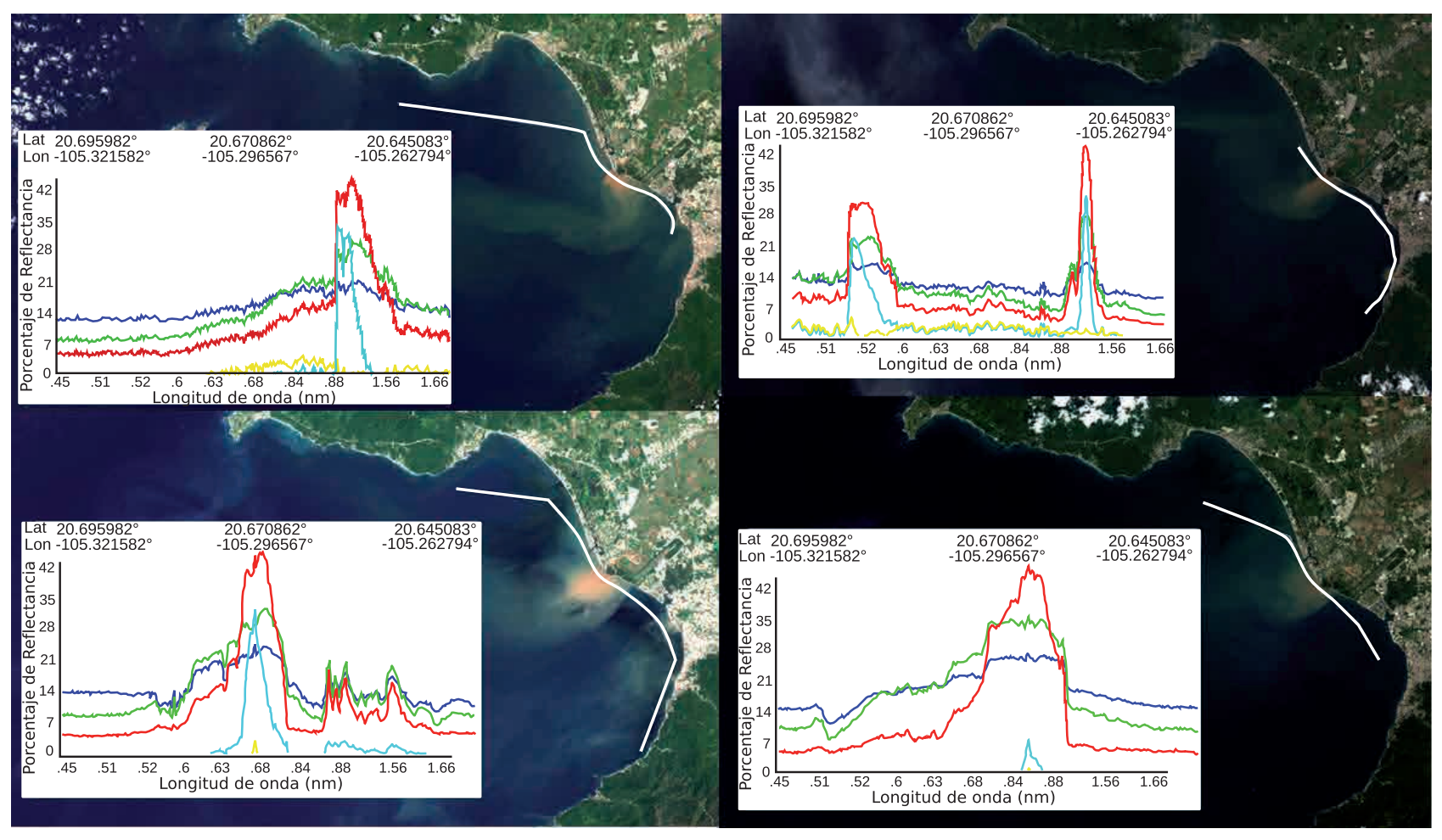

Fig. 4. Transectos en los días (a) 19 de julio, (b) 4 de agosto, (c) 8 de septiembre y (d) 10 de octubre, así como respuesta espectral en la longitud de onda visible (rojo, azul y verde) y de infrarrojos cercano y lejano (cian y amarillo, respectivamente)

\section{CUADRO IV. LONGITUD DE ONDA DE LAS BANDAS VISIBLES E INFRARROJAS DEL SATÉLITE LANDSAT 8}

\begin{tabular}{llcc}
\hline \multicolumn{4}{c}{ Bandas utilizadas en los transectos } \\
\hline $\begin{array}{l}\text { No. de } \\
\text { banda }\end{array}$ & $\begin{array}{l}\text { Porción del } \\
\text { espectro }\end{array}$ & $\begin{array}{c}\text { Longitud de } \\
\text { onda (micras) }\end{array}$ & $\begin{array}{c}\text { colores } \\
\text { utilizados en } \\
\text { las gráficas }\end{array}$ \\
\hline 2 & azul & De 0.450 a 0.515 & azul \\
3 & verde & De 0.525 a 0.600 & verde \\
4 & rojo & De 0.63 a 0.68 & rojo \\
5 & infrarrojo cercano & De 0.845 a 0.885 & rosa \\
6 & infrarrojo medio & De 1.56 a 1.66 & café \\
\hline
\end{tabular}

claridad si la disolución, la sedimentación o algún proceso químico son las razones por las cuales la pluma se hace menos densa.

Gracias a este análisis y al uso de un producto satelital se puede tener una cobertura espacial de toda la bahía con una cobertura temporal representativa de dos o tres días por mes, con la que se puede observar que la tendencia de la dispersión de la pluma de detritos es un movimiento hacia el centro de la bahía con dirección de dispersión hacia mar abierto para las plumas más difuminadas o en posible proceso de sedimentación, y un movimiento marcado hacia el norte para las plumas más densas, las cuales viajas siguiendo la línea de costa.

Se puede observar que en los meses de diciembre a mayo el caudal del río Ameca (a causa de la ausencia de lluvia) es pequeño, lo que se refleja en una densidad preponderantemente media y baja de las plumas de detritos. En este periodo de seis meses, la pluma bordea la costa hacia el norte y su dispersión hacia el centro de la bahía tampoco es muy alta (Fig. 3).

Esta tendencia cambia de forma abrupta a partir de junio y se conserva hasta noviembre, lo cual corresponde a los meses con presencia de lluvias, siendo agosto-septiembre los meses con niveles más altos de detritos vertidos en la bahía. En este periodo de seis meses el caudal vertido empuja a la pluma hacia el centro de la bahía, lo que impide que no corra hacia el norte como pasa en el periodo seco.

La respuesta espectral de la pluma corresponde, en su clasificación más alta (cerca de la salida del río Ameca), a agua turbia con presencia de materia orgánica y algas. Al irse mezclando con el agua de mar y sedimentándose las partículas más pesadas, se observa materia orgánica (clasificación media), 
CUADRO V. LONGITUD DE ONDA DE LAS RESPUESTAS ESPECTRALES VISIBLES E INFRAROJAS DEL AGUA DE MAR CON BAJA CONCENTRACIÓN DE PARTÍCULAS SUSPENDIDAS

\begin{tabular}{|c|c|c|c|c|c|c|c|c|c|c|}
\hline \multicolumn{11}{|c|}{ Agua de mar con concentración baja de partículas suspendidas } \\
\hline \multirow{2}{*}{ Fecha } & \multicolumn{2}{|c|}{ Azul } & \multicolumn{2}{|c|}{ Verde } & \multicolumn{2}{|c|}{ Rojo } & \multicolumn{2}{|c|}{ IR cercano } & \multicolumn{2}{|c|}{ IR medio } \\
\hline & 0.45 & 0.515 & 0.525 & 0.6 & 0.63 & 0.68 & 0.845 & 0.885 & 1.56 & 1.66 \\
\hline $19 / 07 / 13$ & 1900 & 1900 & 1100 & 1100 & 850 & 850 & 0 & 0 & 0 & 0 \\
\hline $04 / 08 / 13$ & 1800 & 1800 & 1000 & 1000 & 800 & 800 & 0 & 0 & 0 & 0 \\
\hline $08 / 09 / 14$ & 1700 & 1700 & 1000 & 1000 & 500 & 500 & 0 & 0 & 0 & 0 \\
\hline $10 / 10 / 14$ & 1100 & 1100 & 900 & 900 & 450 & 450 & 0 & 0 & 0 & 0 \\
\hline
\end{tabular}

CUADRO VI. LONGITUD DE ONDA DE LAS RESPUESTAS ESPECTRALES VISIBLES E INFRARROJAS DEL AGUA DE MAR CON CONCENTRACIÓN REGULAR DE PARTÍCULAS SUSPENDIDAS

\begin{tabular}{|c|c|c|c|c|c|c|c|c|c|c|}
\hline \multicolumn{11}{|c|}{ Agua de mar con concentración media de partículas suspendidas } \\
\hline \multirow{2}{*}{ Fecha } & \multicolumn{2}{|c|}{ Azul } & \multicolumn{2}{|c|}{ Verde } & \multicolumn{2}{|c|}{ Rojo } & \multicolumn{2}{|c|}{ IR cercano } & \multicolumn{2}{|c|}{ IR medio } \\
\hline & 0.45 & 0.515 & 0.525 & 0.6 & 0.63 & 0.68 & 0.845 & 0.885 & 1.56 & 1.66 \\
\hline $19 / 07 / 13$ & 2900 & 2900 & 3000 & 3000 & 2100 & 2100 & 100 & 100 & 400 & 400 \\
\hline $04 / 08 / 13$ & 2500 & 2500 & 2550 & 2550 & 1850 & 1850 & 350 & 350 & 300 & 300 \\
\hline 08/09/14 & 2300 & 2300 & 2400 & 2000 & 1800 & 1800 & 100 & 100 & 0 & 0 \\
\hline $10 / 10 / 14$ & 1500 & 1500 & 1600 & 1600 & 800 & 800 & 0 & 0 & 0 & 0 \\
\hline
\end{tabular}

CUADRO VII. LONGITUD DE ONDA DE LAS RESPUESTAS ESPECTRALES VISIBLES E INFRARROJAS DEL AGUA DE MAR CON ALTA CONCENTRACIÓN DE PARTÍCULAS SUSPENDIDAS

\begin{tabular}{|c|c|c|c|c|c|c|c|c|c|c|}
\hline \multicolumn{11}{|c|}{ Agua de mar con alta concentración de partículas suspendidas } \\
\hline \multirow{2}{*}{ Fecha } & \multicolumn{2}{|c|}{ Azul } & \multicolumn{2}{|c|}{ Verde } & \multicolumn{2}{|c|}{ Rojo } & \multicolumn{2}{|c|}{ IR cercano } & \multicolumn{2}{|c|}{ IR medio } \\
\hline & 0.45 & 0.515 & 0.525 & 0.6 & 0.63 & 0.68 & 0.845 & 0.885 & 1.56 & 1.66 \\
\hline $19 / 07 / 13$ & 2800 & 2800 & 3500 & 3500 & 5500 & 5500 & 3000 & 3000 & 100 & 100 \\
\hline $04 / 08 / 13$ & 2900 & 2900 & 3900 & 3900 & 5500 & 5500 & 2800 & 2800 & 100 & 100 \\
\hline 08/09/14 & 2800 & 2800 & 3500 & 3500 & 5000 & 5000 & 2100 & 2100 & 100 & 100 \\
\hline $10 / 10 / 14$ & 2000 & 2000 & 2700 & 2700 & 3200 & 3200 & 200 & 200 & 0 & 0 \\
\hline
\end{tabular}

preponderantemente. Cuando el agua está más mezclada se puede observar una respuesta que corresponde a clorofila.

\section{CONCLUSIONES}

1. Se puede obtener un producto eficiente y de bajo costo para el monitoreo de plumas de detritos vertidas en bahías con ayuda de imágenes Landsat y Modis si se elimina el ruido espectral que provocan las nubes y la porción continental de las imágenes, y se someten a un proceso de clasificación no supervisada.

2. En el caso de estudio de Bahía de Banderas, las plumas de detritos tienen un empuje hacia la parte central de la bahía en temporada de lluvias y se mueven hacia el norte en la temporada de estiaje.

3. Los resultados presentados no deben considerarse como mapas de contaminantes, ya que este trabajo no tiene la intención de catalogar los tipos de contaminantes presentes en las plumas, sino más bien 
se deben tomar como mapas de vulnerabilidad que hablan de las trayectorias promedio que puede seguir un supuesto contaminante en diferentes épocas.

\section{REFERENCIAS}

Acker J.G., Brown C.W., Hine A.C., Armstrong E. y Kuring N. (2002). Satellite remote sensing observations of storm-induced neritic carbonate transport from shallow carbonate platforms. Intl. Rem. Sens. 23 (14), 28532868. DOI: $10.1080 / 01431160110106122$

Álvarez A.D. y Gaitán J. (1994). Lagunas costeras y el litoral mexicano. McGrawHill, Ensenada, México, 233 pp.

Baumgartner T.R. y Christiensen Jr. (1985) Coupling of the Gulf of California to large-scale interannual climatic variability. Mar. Res. 43, 825-848.

DOI: $10.1357 / 002224085788453967$

Campbell J.B. (2007). Introduction to remote sensing. The Guilford Press, Nueva York, EUA, 432 pp.

Ekercin S. (2007). Water quality retrievals from high resolution IKONOS multispectral imagery: A case study in Istanbul, Turkey. Water Air Soil Pollut. 183, 239-251. DOI: $10.1007 / \mathrm{s} 11270-007-9373-5$

González-de Luna C. (2017). La geomática aplicada al estudio de la dinámica superficial de detritos suspendidos en la Bahía de Banderas. Tesis de Maestría. Posgrado en Hidrometeorología. Departamento de Física, Universidad de Guadalajara, Gudalajara, México, 96 pp.

INEGI-SPP (1983). Carta batimétrica. Isla Revillagigedo, escala 1:1,000,000, cve. CB-006. Instituto Nacional de Geografía y Estadística-Secretaría de Producción Pecuaria.

Kelly G.L., Plata R.L. y Guerrero G.S. (2010a). Predicción de bacterias mediante redes bayesianas en la Bahía de Banderas, México. E-Gnosis 8, 1-27.

Kelly L. (2010b). Variación espacio-temporal de la calidad del agua en la zona de mayor influencia continental en la Bahía de Banderas, México. Tesis de Doctorado. Facultad de Biología, Centro Universitario de la Costa, Puerto Vallarta, México, 129 pp.
Lohrenz S.E., Dagg M.J. y Whitledge T.E. (1990). Enhanced primary production at the plume/oceanic interface of the Mississippi River. Cont. Shelf Res. 10, 639-664. DOI: 10.1016/0278-4343(90)90043-L

Medina-Rosas P. (1997). El efecto de las actividades turísticas sobre los corales pétreos (Cnidaria, Anthozoa, Scleractinia) de Los Arcos, Jalisco, México. Tesis de Licenciatura. Facultad de Ciencias Biológicas, Universidad de Guadalajara, Guadalajara, México, 86 pp.

Miller R.L. y Mckee. B.A. (2004). Using MODIS Terra $250 \mathrm{~m}$ imagery to map concentrations of total suspended matter in coastal waters. Remote Sens. Environ. 93, 259-266. DOI: 10.1016/j.rse.2004.07.012

Pérez B.D. y Nadja O.F. (2011). Comparación de la dinámica de sedimentos suspendidos entre una bahía tropical abierta y una bahía tropical cerrada. Tesis de Maestría. Departamento de Física y Departamento de Geología, Universidad de Puerto Rico, Mayagüez, Puerto Rico, 223 pp.

Pérez O.M. (2011). Aplicaciones de la teledetección y SIG en la caracterización de humedales en la Reserva de la biosfera de la mancha húmeda. Tesis de Maestría. Facultad de Geografía, Universidad Complutense de Madrid, Madrid, España, 132 pp.

Plata L.J. (2007). Dinámica de las ondas internas en la Bahía de Banderas, Jalisco-Nayarit, México. Tesis de Doctorado. Universidad Autónoma de Baja California, Facultad de Ciencias Marinas, Ensenada, México, $152 \mathrm{pp}$.

Plata L., Filonov A., Tereshchenko I., Kelly L., Monzón C., Ávalos D. y Vargas C. (2006). Geostrophic currents in the presence of an internal waves field in Bahía de Banderas, México. E-Gnosis 4, 1-43.

Ritchie J.C. y Cooper C.M. (2001). Remote sensing techniques for determining water quality: Applications to TMDLs. Science Issues Journal 8, 367-374.

Rodríguez G.V. y Gilbes S. (2009). Using MODIS 250 m imagery to estimate total suspended sediment in a tropical open bay. Int. J. Sys. App. Eng. Dev. 3 (1), 36-44. 